\title{
Comments on "Information System? Which One Do You Mean?" by J.A. Carvalho
}

\author{
John Lindsay \\ Reader in Information Systems Design, Kingston University \\ United Kindom \\ Email: lindsay@kingston.ac.uk
}

\section{BROWSING}

\section{ABI, Inspec, ISI, LISA, DAI, URL?}

I could have spent my ten minutes just on the first word of this paper. Where did the browsing happen? What were the terms browsed? "Information", or "systems", or "information and systems"?

If "information" was used, there would be thousands of hits, if "system" similarly, if a logical and then a much smaller number, but the relation between the words and the concepts means that all sorts of stuff might not have been recalled?

If the author didn't use those words in the title of the document, or the abstractor didn't use them in the abstract, or the controlled vocabulary didn't recognise them, then we don't know what would have been retrieved.

In practice the browsing could have been to cast an eye along the bookshelves; in which case if I did the same, there would be some correspondence and much difference.

It says something about the organisation of knowledge in our discipline that we have no shared compendium, collection or encyclopaedia or form of knowledge organisation. 


\section{SYSTEM :: HOLISM}

\section{bus ;; transport ;; city}

The paper discusses first the properties of a system, as a consequence of surveying some of the literature which follows the browsing exercise. There seems to me one important missing concept; that of holism: that the property of a system is that it exhibits a property not present in its components. From the perspective of a designer the important issue is whether this component is the one intended in the design? In other words, does intention produce effect? So a bus is a system which is not just engine, chassis, seats; a transport system is not a conglomeration of buses; and a city is not just a transport system plus housing and employment.

\section{ACTION (TO INFORM) :: ACTION (INTENT, EFFECT)}

The paper goes on to discuss the nature of information and suggests that it is an action (to inform). This seems to me no where near enough. It has to have added to it that the action to inform is with the intent of producing an effect, another action, in the person (or thing) informed, and that information has happened only if the effect is that intended.

\section{IS 1 - 4}

Classical classification

Then is developed a classification scheme in four parts for dividing the authors and possibly their concepts into groups with certain properties.

It would take much more space than here available to consider this with the many others in order to decide their usefulness. Here only to remark that in classical classification we have the concepts of taxis, genera, symmetry, entaxis and parataxis, and we might look at this scheme with that framework.

\section{IST + C + ASSYRIA =/ TD}

The author goes on to suggest what might dangerously approach a technological determinism with his IST, and he leaves out communication, which is not the same as computation, though it might use a computer. In ancient Assyria the land taxation system was build of person, land parcel, good, output, tax level, and the accounts were constructed by reordering the clay tablets. This efficient system had an effect on the society, but deciding the relation between the society and the system gives us the same set of problems we have today. 


\section{EXAMPLES}

FX, Postcode, barcode. UDC, ATA, Read code, Grid reference

There are then a few examples of "systems" and how they fit into the classification. At this point I add a paragraph I didn't say during the discussion for I left a couple of minutes to allow discussion of the list I put here, yet there was none. The list of acronyms at the top I thought some people would know and others not, but this list I suspected contained some which would be uncommon. From the absence of question I draw a conclusion.

\section{KM, TQM, BPR, BSE}

Then there is a discussion of frameworks like FRISCO, which have been fashionable at various stages to which perhaps could be added knowledge management, total quality management, perhaps Checkland, UML, XML, Zackman, SSADM, RAD, DSDM, Multiview to see whether the classification stands up?

We seem still to me to be addressing the problem of whether information systems design has information as the dominant planet with systems, science, management, etc as moons; information and systems as dipoles or logical ands; system as the planet with information as a moon along with transport, logistical, operational, etc; or information systems as a new thing in the way water does not contain the properties of hydrogen and oxygen.

It is what I call our version of BSE, mad cow disease: do we regard information systems design as a business, a science or engineering, and of which do we use the proof protocols? 